\title{
ECOSISTEMA GRÁFICOS. Un paso más desde el grabado no tóxico para la Agenda 2030 y los Objetivos de Desarrollo Sostenible.
}

\author{
Tomás-Miralles, Ana ${ }^{a}$; Ansio-Martínez, Tania ${ }^{\mathrm{b}}$ y Aguilar-Briceño, Rosángela ${ }^{\mathrm{c}}$ \\ ${ }^{a}$ Títular de Universidad de la Facultad de Bellas Artes de la Universitat Politècnica de València e investigadora del \\ Centro de Investigación Arte y Entorno CIAE, atomas@dib.upv.es, ${ }^{b}$ Licenciada en Bellas Artes y Doctoranda en la \\ Facultad de Bellas Artes, Universitat Politècnica de València, taniasab@gmail.com y Rosángela Aguilar Briceño ${ }^{c}$ \\ Graduada en Bellas Artes por la Universitat Politècnica de València vinculada y colaboradora del Centro de \\ Investigación Arte y Entorno CIAE, rosangelaaguilarb@gmail.com.
}

\section{Abstract}

Etching as a graphic method is not only a set of techniques, it also has the scope of a SYSTEM with all that this implies. And as a system, the education of these subjects is directly linked to the fulfillment of the SDG's 2030, by focusing on the various objectives that are set from an ECOSYSTEM perspective: A form of art practice resorting to its ease of multiple reproduction as a matter of social approach and by constant experimentation on classical and new techniques. The transmission of our graphic approaches is immersed in public awareness of the importance of planet conservation and sustainability, thus motivating artistic practice in the context of becoming aware of the ecological impact of human activity. We are all called upon to act from the new culture of sustainability that permeates everything and is decisive. In experimentation classrooms, the joint of our team and out students at the UPV, has eased the path to develop our projects BOSQUEARTE and MONTA TUS OBJETIVOS, in order to raise awareness, throughout the workshops and art happenings that surrounds them, with the need to commit ourselves to preserving the environment and natural resources.

Keywords: Sustainability, Environment, Culture, Raising Awareness, Graphics, Creation, Non-toxic Etching.

\section{Resumen}

El Grabado como método gráfico no es sólo un conjunto de técnicas, sino que tiene la envergadura de un SISTEMA, con todo lo que ello supone. Y como sistema, la educación de estas materias se vincula directamente con el cumplimiento de los ODSs 2030, focalizando los distintos objetivos que nos planteamos desde una perspectiva de ECOSISTEMA: una forma más de práctica artística, acudiendo a su facilidad de reproducción múltiple como una cuestión de acercamiento social y por la constante experimentación sobre las técnicas clásicas y las de nueva aparición.

La trasmisión de nuestros planteamientos gráficos está inmersa de la conciencia pública sobre la importancia de la conservación del planeta y de la sostenibilidad, motivando así la práctica artística en el contexto de toma de conciencia del impacto ecológico de la actividad humana. Todos estamos interpelados a actuar desde la nueva cultura de la sostenibilidad que lo impregna todo y es decisiva.

En aulas de experimentación, con el equipo y los alumnos de los talleres de la UPV, hemos desarrollado parte de nuestros proyectos BOSQUEARTE y MONTA TUS OBJETIVOS, para sensibilizar, a través de talleres y/o acciones artísticas, sobre la necesidad de comprometernos con preservar el entorno y los recursos naturales.

Palabras clave: sostenibilidad, entorno, cultura, concienciación, gráfica, creación, grabado no tóxico. 


\section{Introducción}

La docencia de una asignatura como el Grabado no puede entenderse sólo como el aprendizaje de un conjunto de técnicas reproductivas, sino como un SISTEMA con todo lo que supone considerar la envergadura de un sistema y sus ramificaciones. Como sistema, puede ser trabajado desde cada una de sus partes para focalizar en ella los distintos objetivos que nos planteemos.

Nuestros proyectos BOSQUEARTE y MONTA TUS OBJETIVOS, surgen de ésta posibilidad y, al igual que un organismo vivo, se fundamentan y ramifican para responder a la emergente llamada social. Nuestros planteamientos gráficos están inmersos de la conciencia pública sobre la importancia de la conservación del planeta y de la sostenibilidad, situando así la experimentación de la práctica artística en el contexto de toma de conciencia del impacto ecológico de la actividad humana. Todos estamos interpelados a actuar porque la nueva cultura de la sostenibilidad lo impregna todo. El camino a seguir transita por el cumplimiento de los ODSs 2030.

Desde siempre artistas y colectivos culturales reconocen en el Grabado una magnífica forma de práctica artística de carácter social y educativo, acudiendo a su facilidad de reproducción múltiple como a una cuestión de acercamiento social y por la constante experimentación sobre las técnicas clásicas y las de nueva aparición. Hoy en día disponemos de amplios y mejores recursos científicos y logísticos para tener un impacto positivo en la concienciación del cumplimiento de los Objetivos de Desarrollo Sostenible de 2030.

Esto nos proporciona la ventaja de desarrollar una variedad de posibilidades artísticas que estén enfocadas en innovar en la educación universitaria.

Desde la asignatura de Grabado, nuestra experiencia es que puede desarrollarse transversalmente con el alumnado el ejercicio de concienciar, a través de talleres y/o acciones artísticas, sobre la necesidad de comprometernos en la preservación del entorno y los recursos naturales.

Con el sistema gráfico desarrollado a partir de la experiencia con Bosquearte y monta tus Objetivos, descubrimos que, desde el aula, tenemos un papel crucial que desempeñar en el desarrollo y aplicación de nuevas técnicas de grabado sostenibles, que promuevan una transición positiva a una creación artística más consciente con el medioambiente.

Ponemos nuestro granito de arena para configurar este ECOSISTEMA GRÁFICO que, desde la experimentación con la práctica artística, moviliza a la sociedad, repensando nuestra manera de vivir en este mundo, replanteándonos que podemos mejorar en la técnica artística y aceptando nuestra responsabilidad hacia las generaciones venideras para que ello se traduzca en una educación consciente.

\section{Objetivos}

Desde la investigación artística se pretende enfocar dentro del desarrollo curricular, en algunas de las asignaturas de los talleres de Grabado de la Universitat Politècnica de València, hacia aulas de experimentación para el activismo artístico, a cargo de la profesora Ana Tomás Miralles y su equipo de especialistas, en torno a los siguientes objetivos:

- Difundir la conciencia ambiental a través de la generación de sinergias en creación artística

- Promulgar la experimentación en Gráfica en las aulas y/o talleres de arte universitario

- Generar compromiso con la preservación del entorno y los recursos naturales 
- Emprender en el desarrollo y aplicación de técnicas de grabado no tóxico de cara a los Objetivos de Desarrollo Sostenible de la Agenda para 2030.

\section{Desarrollo de la innovación: Educar para concienciar}

El desarrollo de la experiencia está sustentado en los siguientes focos de interés:

- Proyecto Bosquearte: Crear para cambiar

- Proyecto Monta tus objetivos: Tú formas parte del camino a la sostenibilidad

- Ecosistema Gráfico, con el registro de la huella incisa y la huella excisa

- Técnicas y tratamientos de la Gráfica Sostenible

- Trabajo en Equipo para enfatizar, reflexionar y concienciar

- Consigna de las tres erres Ecológicas: respetar, recuperar, reivindicar

Desde el ecosistema gráfico así entendido, se pretende promover la conciencia medioambiental para cultivar la curiosidad estableciendo relaciones entre el aula de clases y los proyectos educativos que les suscitan, de los que aquí se viene a profundizar.

Estos vínculos se entretejen en torno a la coincidencia en objetivos e intereses para sensibilizar a la sociedad y fomentar la participación. Cada uno en su campo, busca la forma de Enfatizar, Reflexionar y Concienciar para la transformación cultural.

\subsection{Proyecto Bosquearte}

Los objetivos que se persiguen con el proyecto Bosquearte devienen del compromiso con el entorno y los recursos naturales. Como titula el artículo de Lucía Vázquez García (2018) en Emocionar, inspirar y educar a través del Arte, "el arte se erige como una potente herramienta para visibilizar el cambio climático y la sostenibilidad. Un arma que integra ciencia, tecnología o filosofía y que abarca lo local, lo regional, nacional e internacional". Por ello para contextualizar la innovación propuesta, se entiende en forma y fondo la necesidad de transmitir el mensaje a la sociedad. La subjetividad y multiplicidad de significados aportados por la creación artística permiten indagar en un tema tan complejo como lo es el cambio climático, al hacerlo accesible a todo público desde la didáctica y la plástica, desde el aprendizaje visual.

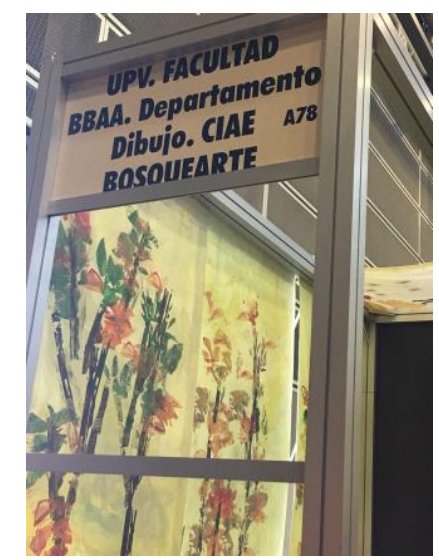

Fig. 1: 2018, noviembre. Stand Bosquearte en IBERFLORA, la Feria Internacional de planta, flor, paisajismo, tecnología y bricojardin de Valencia. 
El proyecto base, nació inicialmente de la colaboración con una escuela infantil en 2012. Desde entonces hemos hecho trabajos colaborativos fusionando y generando epistemología. Éste ha ido desarrollándose a lo largo de los años como un complejo sistema gráfico, constituido por casi un centenar de exposiciones, numerosos talleres y clases magistrales sobre el grabado no tóxico. Planteándose inicialmente desde lo didáctico y lúdico, hoy en día pasa a ser un ambicioso proyecto en aulas de experimentación, que procura descubrir medios alternativos de grabado sostenible que promuevan los métodos de enseñanza en Gráfica y ayuden a la visibilización de otras realidades.

Su objetivo es propiciar aprendizajes que se fijen en el conocimiento, mediante prácticas coherentes con la realidad actual. La intervención que surgió en primera instancia en dos ámbitos como son la Escuela y la Universidad, ha descubierto estrategias didácticas en distintos sentidos y niveles.

Desde este punto de vista, responde este proyecto a la exigencia recogida en el Plan Bolonia de integrar la docencia en un terreno globalizador de investigación, innovación y el desarrollo $(\mathrm{I}+\mathrm{D}+\mathrm{I})$, que puede ser considerado como la regla de juego que Ibarra Collado denomina capitalismo académico, con la capacidad de imaginar escenarios distintos que nos permitan edificar un sistema de producción de conocimiento que atienda las necesidades de la sociedad y no sólo aquellas de la economía (Slaughter y Leslie, 1997). Ello implica la incidencia en cadena hacia la validación de una economía más sostenible y respetuosa con el medio ambiente.

Se trata de motivar en los alumnos una puesta en valor del uso consciente y concienciado de los métodos, procesos y técnicas de la asignatura (en la eliminación de productos tóxicos), así como aproximar al público hacia estas experiencias artísticas revalorizando el entorno que nos circunda, y destacando el buen uso de los recursos naturales, culturales y paisajísticos.

En The Hangzhou Declaration de 2013, publicada por la Organización de las Naciones Unidas para la Educación, la Ciencia y la Cultura, se afirma:

"Value, safeguard and transmit culture to future generations Heritage is a critical asset for our well-being and that of future generations, and it is being lost at an alarming rate as a result of the combined effects of urbanization, development pressures, globalization, conflicts and phenomena associated with climate change." (p.8) (Valorar, salvaguardar y transmitir la cultura a las generaciones futuras el patrimonio es un activo fundamental para nuestro bienestar y el de las generaciones futuras, y se está perdiendo a un ritmo alarmante como resultado de los efectos combinados de la urbanización, las presiones de desarrollo, la globalización, conflictos y fenómenos asociados con el cambio climático.) Así pues para proteger estos activos, es decir los ecosistemas naturales, se precisa la transmisión de valores y/o éticas ecológicas que involucren la salvaguarda como modo responsable de vida perdurable en el tiempo.

Por eso, entre los objetivos que se buscan en los proyectos, compartimos con los alumnos y los demás participantes la cultura de la sostenibilidad, que se fundamenta en favorecer actitudes positivas, fomentar la solidaridad, y la empatía para la percepción del mundo natural y promoviendo así el acercamiento social.

Bosquearte ha ido vertebrándose en diferentes direcciones y en él, incidimos en parte de los objetivos de la Agenda 2030 para el Desarrollo Sostenible, un plan de acción a favor de las personas, el planeta y la prosperidad que adoptó la Asamblea General de la ONU en septiembre de 2015.

Relatar todo el proyecto sería complicado por su envergadura actual. A lo largo de ocho años, ha ido afianzándose como un evento de carácter divulgativo y expositivo que tiene su momento cumbre cada año en Iberflora, Salón del árbol de la Feria de Muestras de Valencia, integrándose en jornadas internacionales de gráfica y arboricultura, y con exposiciones nacionales e internacionales. 
Las exposiciones, talleres, performances, mesas redondas y otros eventos paralelos de obra gráfica que se presentan, abordan en toda su extensión y variantes aspectos relacionados con la temática medioambiental, biodiversidad y sostenibilidad en su expresión más global. Asimismo, en el desarrollo de las propuestas, se hace alusión a las tendencias actuales gráficas que tienen como objetivo dialogar con el público en relación a los Objetivos de Desarrollo Sostenible 2030.

La iniciativa pretende aportar diferentes discursos y puntos de vista con el objetivo de llevar a cabo una invitación a la reflexión sobre nuestro entorno, su génesis y crecimiento, promoviendo a través de la imagen y el lenguaje expresivo-plástico, la biodiversidad.

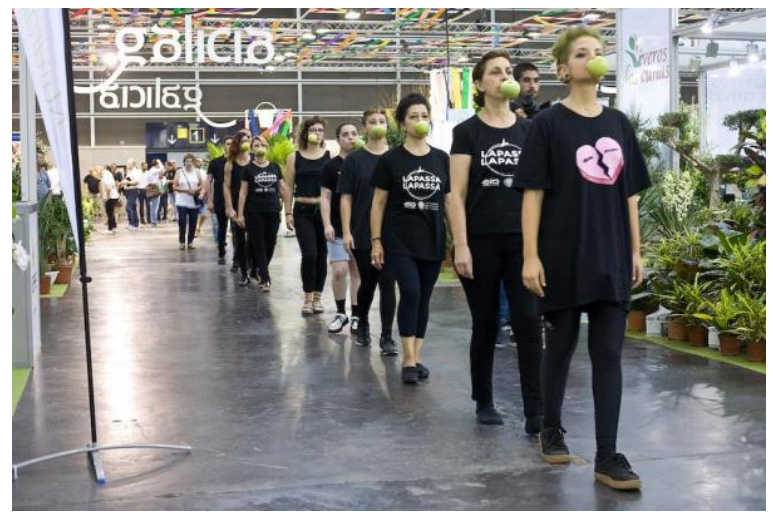

Fig. 2 : 2018, noviembre. Performance La Passa en IBERFLORA, la Feria Internacional de planta, flor, paisajismo, tecnología y bricojardín de Valencia.

A través de diferentes planteamientos artísticos, se propone con los alumnos y colectivo implicado, situar la expresión artística y cultural como recurso al servicio de la sociedad, ayudando a aportar visibilidad ante diferentes líneas de transformación cultural en pro de modelos de sociedad más sostenibles. Asimismo, se propone contribuir a la empatía con el medio en esta época antropocena: la era del impacto del ser humano en la Tierra.

Para desarrollar positivamente el proyecto es necesario un plan estratégico, táctico y eficiente. Orientar y dirigir todos los proyectos de talento artístico personales, así como los procesos de gestión institucionales, aplicar un modelo de calidad académica para con el colectivo, y trazar metodologías activas que permiten una solución coherente y provechosa.

\subsection{Proyecto Monta tus objetivos}

En 2018 la Generalitat Valenciana a través del Vicerrectorado de Responsabilidad y Cooperación Social de la Universitat Politècnica de València convoca al premio de Campaña de difusión los ODSs, otorgando el primer lugar a nuestro equipo, el grupo ATOTARO, Diversidad Gráfica, compuesto por Ana Tomás, profesora titular de la UPV; Toni Simarro, Técnico Superior de Investigación y adscrito al Centro de Investigación Arte y Entorno (CIAE); Tania Ansio, doctoranda en la Escuela de Doctorado de la UPV y Rosángela Aguilar, Graduada en Bellas Artes y colaboradora del Centro de Investigación Arte y Entorno (CIAE), con el proyecto Monta tus objetivos: TÚ formas parte de la cadena hacia la sostenibilidad, que difunde y promueve el conocimiento sobre los ODSs a través de la participación de la comunidad universitaria.

El proyecto Monta tus objetivos en estrecha relación con las 5 Universidades Públicas de la Comunidad Valenciana (Universidad de Alicante UA, Universidad Jaume I de Castellón UJI, Universidad de Valencia UV, Universitat Miguel Hernández de Elche UMH y la Universitat Politècnica de València UPV), educa 
sobre cada uno de los 17 ODSs para 2030 a través de sus signos identificativos tanto en color como en forma, por medio de talleres artísticos de xilografía llevados a cabo en cada una de ellas y por medio de la difusión de los mismos en redes sociales, generando miles de impresiones y variadas interacciones entre los internautas de la comunidad universitaria.

Para la organización y realización de este proyecto se cuenta con la colaboración de los alumnos de grabado, que responden al aprendizaje a través de la experiencia de llevar a cabo un proyecto de esta envergadura en la Comunidad Valenciana a través de la participación de la comunidad universitaria. Este incluye un aprendizaje activo en la que la colaboración es vital.

Esta difusión se lleva a cabo mediante 5 workshops de estampado xilográfico, sobre papel y camisetas en las 5 Universidades de la Comunidad Valenciana, en cuya puesta en práctica colaboran la profesora, su equipo y los alumnos de la asignatura.

Se parte del hecho de que la práctica artística es un magnífico medio para resituar y encauzar un modelo de ciudadanía comprometido activamente con la consecución de un mundo más equitativo y sostenible. Siguiendo esta premisa, desde el arte y el sistema gráfico, se exterioriza e instruye en colectividad el papel crucial que tenemos que desempeñar en las aspiraciones universales de progreso humano, económico, social y ambiental.

En los talleres se trazaron dinámicas de impresión de matrices xilográficas siguiendo los lineamientos de cada ODS e informando a los participantes de la definición de cada uno, así pues, se establecen conexiones teórico-plásticas entre el concepto y la imagen de los ODSs 2030.

También se trabajan estos conceptos con la técnica xilográfica elegida, que deviene de la creación de matrices modeladas en madera reciclada con la innovativa técnica de corte láser en los laboratorios del Departamento de Dibujo de la Facultad de Bellas Artes de la Universitat Politècnica de València. Dichos soportes son entintados con tintas no tóxicas, elaboradas con base de aceites vegetales y pigmentos naturales, en vez de utilizar tintas procedentes de hidrocarburos.

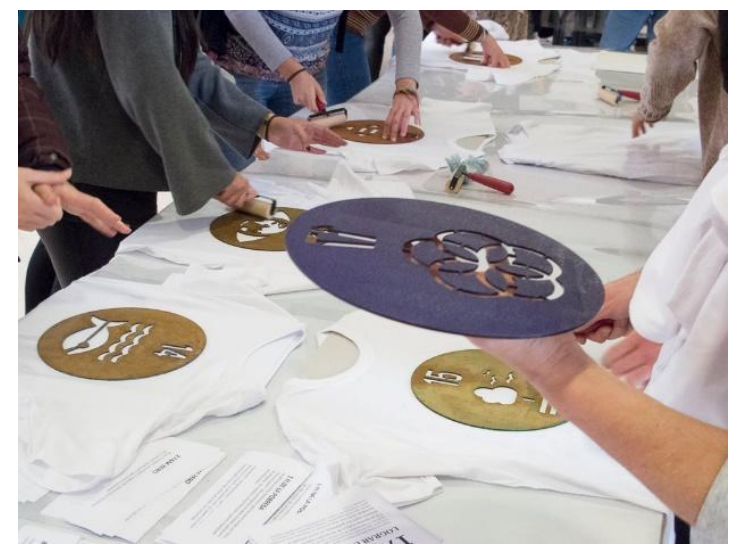

Fig. 3. 2019, marzo. Universidad de Alicante. 4to taller Monta tus objetivos. Estampación xilográfica de tinta no-tóxica sobre tela

El aprendizaje de una técnica gráfica en torno a un objetivo medioambiental forma al alumnado de la línea gráfica y lo mueve a compartir la experiencia junto con el resto de alumnos de otras universidades, incrementando el aprendizaje y la motivación. La obra resultante pasa a ser objeto de difusión: La imagen plasmada se convierte en icono rehumanizado, alterado por la mano del participante, concediéndole así más posibilidades de interpretaciones subjetivas y plásticas. Monta tus Objetivos crea, de esta manera, nuevas 
vías de construcción de la imagen estampada, usando la educación como motor de aprendizaje-difusión de las metas medioambientales a alcanzar.

Estableciendo la participación de diferentes agentes académicos en materia de educación y mediante el programa de cooperación se ha conseguido una implicación y participación mayor de lo esperado inicialmente.

Bajo el paraguas de la creatividad plástica y artística generamos la idea de una performance artística, lográndose talleres que alcanzan la categoría de eventos artístico-participativos, en la que las estampas de los ODS se convierten en núcleos de información y enseñanza, así como en piezas performativas colocadas en modo de póster, de estructuras, de instalación.

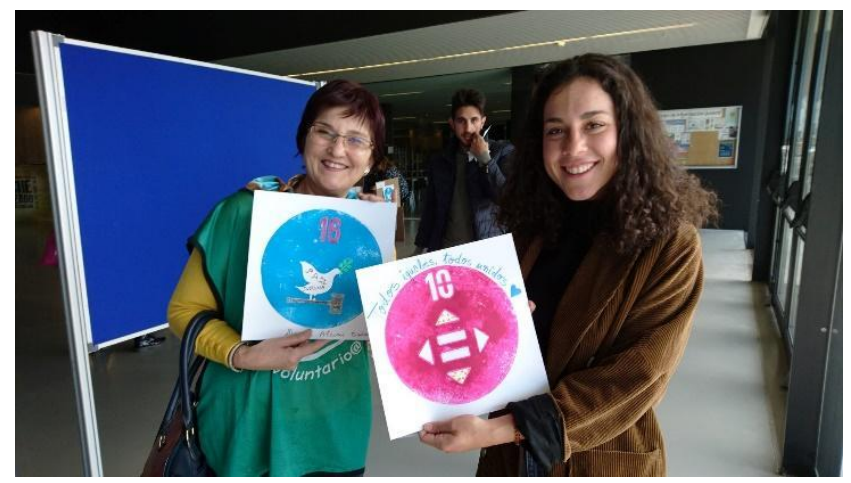

Fig. 4 : 2019, abril. Taller Monta tus Objetivos. Universidad Miguel Hernández de Elche. Obra resultante

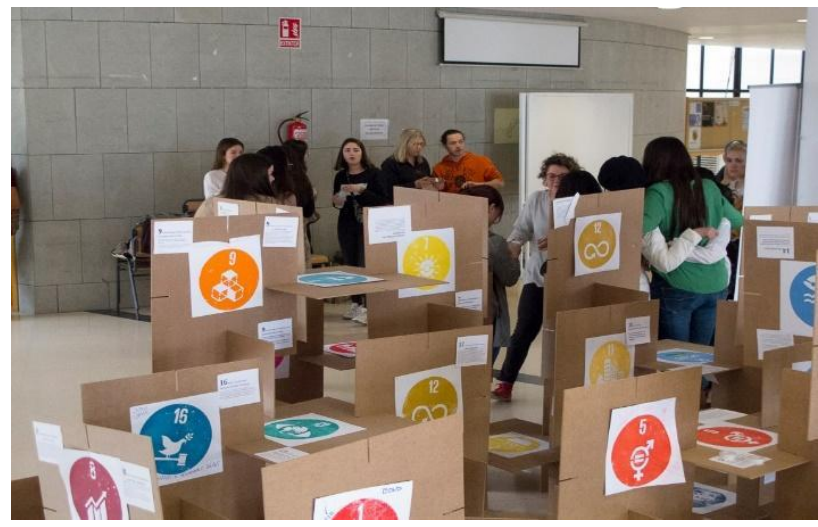

Fig. 5 : 2019, febrero. Universitat Politècnica de València. Objetivos montados en modo de estructura por parte de los alumnos.

\subsection{Técnicas y tratamientos de los proyectos desde la Gráfica sostenible.}

Desde el primer momento se articulan ambos Proyectos con criterios de Gráfica Sostenible, con un espíritu creativo que potencia y estimula en los alumnos artistas las capacidades emocionales buscando producir la toma de conciencia. Como dice María Novo: "Concienciar a la población sobre estos temas, estimular la necesidad del cambio, construir alternativas en las mentes y el corazón de los habitantes del planeta es tarea de la educación" (Novo, 2017). Dichas dinámicas son construidas por medio de la práctica directa, la estimulación y el intercambio de ideas, y facilitando el crecimiento personal e intelectual de los individuos que de ellas sean participantes proactivos. 
Para promover estilos y disciplinas artísticas sostenibles, desde el conocimiento holístico del taller se busca la concienciación de los participantes en las técnicas de Grabado menos tóxico en técnicas y tratamientos, pues las técnicas gráficas tradicionales, se caracterizan por ser sumamente contaminantes, tanto en las fases de elaboración de matrices como en su posterior desarrollo a la estampa, cuestión mitigada en los últimos tiempos con la puesta en marcha de la fase de recuperación de ácidos y productos tóxicos por empresas contratadas desde la UPV, dirigidas desde el Departamento de Dibujo y el técnico del laboratorio Jonay Cogollos.

En la experimentación desde el aula para llevar a cabo estos proyectos: IBERFLORA y MONTA TUS OBJETIVOS, nos planteamos llevar a cabo una tarea consciente de reducirlos al máximo, minimizando la huella ambiental negativa generada en su proceso.

Así se promueve el uso de productos biodegradables, tintas no contaminantes, el procesamiento de las matrices con mordientes y técnicas más sostenibles, y reducir los residuos tóxicos reemplazando esencias provenientes de hidrocarburos como la esencia de petróleo con el uso de aceites vegetales.

No sólo se trata de hablar de un tema y concienciar a la sociedad, se trata de que, desde el aula-taller, se entienda que este cambio social ha de comenzar en el punto de creación mismo.

Por ello, parte de este proceso de concienciación es resignificar también el uso de productos perjudiciales para el medio ambiente como lo son aquellos provenientes de los hidrocarburos, el petróleo y su más corrosivo derivado: el plástico y sus micropartículas, gran contaminante del agua y los ecosistemas, conforme a The Hangzhou Declaration.

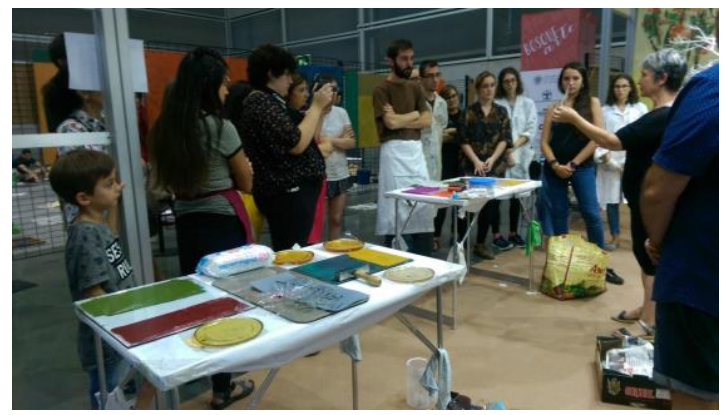

Fig. 6. 2017, noviembre. Taller-exhibición por Tania Ansio: "Otoño gráfico" en IBERFLORA, la Feria Internacional de planta, flor, paisajismo, tecnología y bricojardín de Valencia.

Se tiene en cuenta entonces, que un uso de técnicas sostenibles en las aulas de clase, promueve no sólo un ambiente más salubre para el alumnado y genera conciencia ambiental, sino que promueve a mayor escala avances en el desarrollo de protocolos de taller más empáticos con el medio ambiente y con la política de separación sostenible de desechos en el ámbito universitario. Es decir, se crea responsabilidad y compromiso frente a los residuos generados.

Contamos también con el soporte que nos ofrece la Unidad de Medio Ambiente de la Universitat

Politècnica de València bajo la supervisión de Jonay Cogollos desde el aspecto técnico y la gestión. 


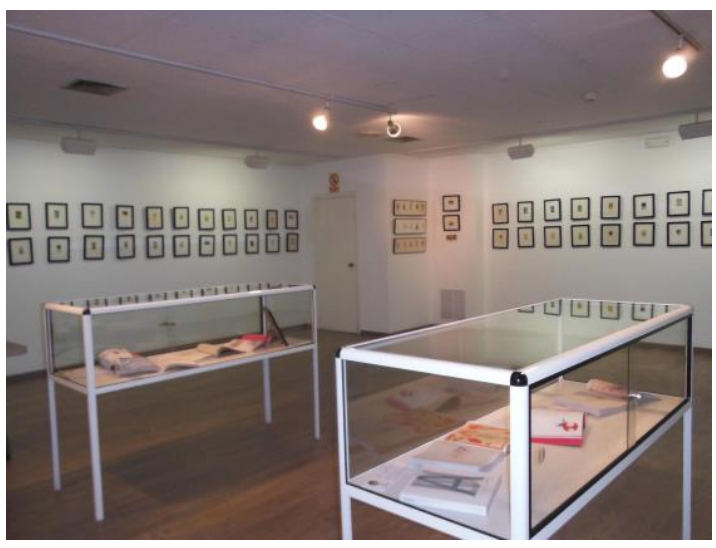

Fig. 7: 2015, marzo. Casa de la Cultura de Quart de Poblet. Exposición: Bosquearte:AdentrArte en un bosque ilustrado.

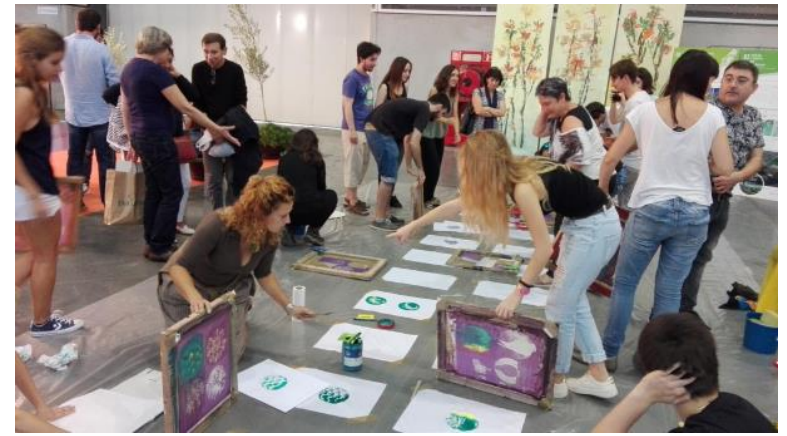

Fig. 8. 2016, septiembre. Performance Facultad BBAA, UPV en IBERFLORA, la Feria Internacional de planta, flor, paisajismo, tecnología y bricojardín de Valencia.

\subsection{Herramienta visual de los proyectos: Exposiciones.}

El trabajo en equipo desarrollado en el taller para ambos Proyectos tiene su culminación en el hecho expositivo.

Las exposiciones en sí mismas, constituyen un medio muy potente de comunicación y de generación de conocimiento. Son el momento en que el artista o el grupo presenta en sociedad el trabajo realizado.

Los alumnos-creadores tienen en ese momento la posibilidad de aportar sus consignas poéticas sensibles y expresivas a través de la experimentación gráfica. Están con una capacidad de crear y potenciar estrategias que generen nuevas visiones de la sociedad deseable a la que aspiramos.

Consideramos que el arte se puede manifestar como un privilegiado espacio de creación de conocimiento sobre el mundo en que queremos participar de forma activa, de desarrollar los modelos que se consideran adecuados a los proyectos, con la necesidad urgente de generar espacios de discusión que muestren una posibilidad de transformación cultural y busquen una realidad diferente a la que estamos viviendo, una más sostenible.

Las exposiciones planteadas por ambos Proyectos incluyen la muestra de obra gráfica realizadas tras la impartición de la materia o tras el desarrollo de talleres.

El extenso sistema de trabajo en equipo planteado incluye: Obra Gráfica, Expresión y creación visual, Exposiciones interactivas de aproximación, Valores ecológicos, Dibujos, Performances, Mesas Redondas entre muchos otros. 
Es igualmente de gran relevancia la iconografía utilizada en la producción de las obras. La generación de conciencia a través de la imagen constituye un medio resignificante de la materia impresa.

En el proyecto MONTA TUS OBJETIVOS, las claves de dicho lenguaje son muy claras al abarcar los iconos correspondientes a los ODSs 2030 así como su asociación por color, abriéndose como alternativa el juego de alternancia plástica del color frente a los símbolos.

Por otro lado, el proyecto BOSQUEARTE pretende jugar con el imaginario colectivo, de la consciencia, volcando sus directrices en estampaciones de lenguajes gráficos que se asemejan a las texturas presentes en la naturaleza. Imágenes por asociación: Naturaleza, biosfera, clima, flora, fauna e interacciones humanonaturaleza.

Así pues, unificamos en nuestros participantes la conciencia de Ecosistema Gráfico, pues, el medio menos tóxico adquirirá una resignificación positiva tanto a nivel semántico, como plástico.

La carga de implicación que ha habido en los últimos años se puede cuantificar de algún modo con las actuaciones desde diferentes colectivos: En primer lugar, cada curso, los aproximadamente 90 alumnos de diferentes asignaturas de gráfica: unos 45 alumnos de cada grupo y dos grupos cada año, uno por cada semestre. Otro de esos enriquecedores grupos ha sido el de los miembros del Centro de Investigación Arte y Entorno CIAE, de la Facultad de Bellas Artes de la Universitat Politècnica de València; también el grupo Lapassa de Generación Espontánea de la UPV; en otras ocasiones participa la escuela infantil Príncipe Valiente y el instituto Pere Boïl o los grupos: "Compromiso Gráfico" con Inma Peiró, Tania Ansio, Ana Tomás y Javier Esquerdo; "Gráfica Biosensible” con Rosángela Aguilar, Tania Ansio y Ana Tomás; "Diversidad Gráfica" con Tania Ansio, Toni Simarro, Rosángela Aguilar y Ana Tomás

Estos agentes han formado parte y seguirán formándola de algunos de los eventos comunes, así como los acontecidos en Iberflora, desde el año 2016.

Las diferentes actuaciones se pueden cuantificar con el número y título de las exposiciones, que hacen un total de aproximadamente 85 muestras colectivas donde participan los alumnos como creativos o como observadores. Con esta temática se ha intervenido desde 2012 en eventos internacionales, nacionales y locales de la misma línea de investigación, no todos protagonizados por los alumnos pero que sí han podido visitar o participar en su montaje, lo que les suscita máximo interés y preocupación por la obtención de óptimos resultados personales.

Como muestra, algunos de los títulos de exposiciones realizadas han sido los siguientes:

ConectArte con el medioambiente, SaludArte, EntornArte en la naturaleza, CamuflArte en la estampación, EnredARTE entre grafismos, AdentrARTE en procesos creativos, EstampARTE: Surcos y huellas, ¿Bos Qué?, Impresión-Expresión, Oxigenarte, RamificArte, EnlazArte, Al bosque a buscarte, Pequeña "estampa" de un futuro bosque, La Huella incisa del Bosque en el Arte-La Huella excisa del Arte en el Bosque, Naturaleza fotosensible, BiodegrabArte, Ecosistemas naturales de la gráfica, $\mathrm{H} 2 \mathrm{OH}$, lemas y discursos sostenidos-bles, Ecoidentidad, ArborYcultura, Gráfica biodiversa, biocenosis marina, Sangra el mundo, plantarte ideas, Biodiversidad arbórea, de hoy no pasa-tienes que plantArte, Endinsar-te en les petjades gràfiques, pasión por el verde, plantarte ideas, biotopo gráfico: reflexión artística sobre el impacto medioambiental, por una Europa Sostenible, Mother nature, quejidos de la Tierra, Arborízate, Art amb la ciència i a consciència, Semilla de Esperanza, naturaleza fotosensible, bosque a través del Arte, Naturaleza articulada-Naturaleza divergente, Naturaleza oceánica-Naturaleza acuática, Naturaleza sostenidaNaturaleza rural, Naturaleza enturbiada-Naturaleza entrapada, Naturaleza reprogramada-Naturaleza agredida, ¿Naturaleza de plástico o Naturaleza de plancton?, Naturaleza recuperada-Naturaleza copiada, 
Naturaleza velada-Naturaleza protegida, Naturaleza evaporada-naturaleza vacía, El grito-la naturaleza desierta, etc.

Con la asistencia a estos eventos la formación fuera del aula se ve incrementada, pisando el terreno y competitividad real. Parte de la base que sobremotiva a los alumnos de la línea de gráfica en su trabajo de taller, es el saber que su estampa va a poder mostrarse al público y va a tener una evaluación que se reflejará en su nota final.

El poder participar como invitados en eventos de reconocido prestigio guiados por su profesora es una cuestión poco frecuente mientras cursan la carrera. El discente sabe muy bien que no todos los profesores favorecen el acercamiento a la profesionalización real: eso marca una pequeña diferencia. Que un trabajo académico pueda exponerse, hace que el desarrollo del mismo se vea desde diferentes puntos de vista, se trabaje más a gusto y acabe siendo más competente que otros ejercicios de igual calado. Ellos saben que tienen que aprovechar las circunstancias y tienen que dar todo lo que han aprendido por ellos mismos para que sus visualizaciones puedan derivar en reflexiones del público en torno al tema y en torno a la calidad procesual, compositiva, cromática, gestual, lineal, etc.

Para ello, prácticamente todo el alumnado se acoge a la propuesta de la profesora sobre temática, tamaño y técnica que viene determinada para unificar argumentos y profundizar en los mensajes que se quieren transmitir a la sociedad. De esta forma, se potencian los estímulos formativos de cada participante, aprenden a gestionar mejor sus tiempos, sus energías, el acabado final y no olvidan la faceta de puesta en escena fuera del territorio universitario.

\section{Conclusiones y Resultados}

Coincidimos con los participantes del congreso IN-RED 2020 en nuestro compromiso con una educación de innovación que promueva los ODS 2030, desde nuestra área que es la creación y la técnica gráfica.

En los proyectos aquí presentados, se establecen vínculos transversales para enfatizar, reflexionar y concienciar desde nuestra realidad educativa e investigadora en la preservación del entorno y los recursos naturales.

En el aula, con los alumnos de la asignatura de grabado, se potencian los estímulos formativos de cada participante, se les educa en gestionar mejor sus tiempos, sus energías, el acabado final y se les facilita la faceta de puesta en escena fuera del territorio universitario. Es muy interesante poder enmarcar a los futuros profesionales en el espacio de los trabajos amateurs, para que tomen impulso hacia esa profesionalidad. Con estas propuestas se busca producir imágenes que favorezcan la valoración de nuestra propia conducta de cara al medioambiente, problematizar la evidente realidad sobre el cambio climático, y favorecer al imaginario colectivo a través de la difusión de actividades artísticas, planteando sinergias entre la conciencia ambiental, la participación y el activismo, redirigiendo la actividad humana hacia vías ecológicamente sustentables y éticas, y con un impacto beneficioso para el desarrollo de una cultura de la sostenibilidad.

Los alumnos y colaboradores son conscientes de la generación de un cambio social en el que nuestra colaboración como artistas vendrá del camino de promover escenarios expositivos y de la divulgación de resultados y comunicación de nuestra participación con el empleo de técnicas menos tóxicas, para que se posicionen como alternativa frente a las procedentes de hidrocarburos. 


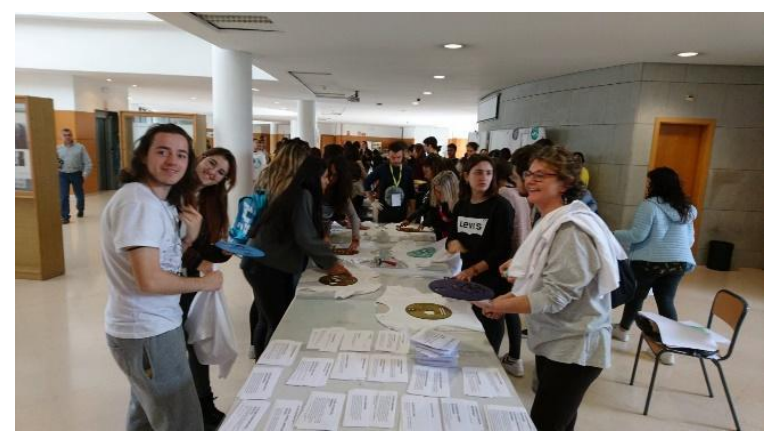

Fig. 9. 2019 marzo. Universidad Miguel Hernández de Elche. Desarrollo del taller.

En las aulas de la asignatura de la Facultad de Bellas Artes, nos hemos propuesto innovar siguiendo parámetros teóricos y prácticos, pues los alumnos a la par que aprenden las técnicas propias de un SISTEMA gráfico, desarrollan otra experimentación y procesos que, como un ECOSISTEMA, favorecerá un mosaico de posibilidades artísticas a desarrollar y buscará constantemente encontrar un impacto positivo en sus personas y en la sociedad.

Con los proyectos BOSQUEARTE Y MONTA TUS OBJETIVOS hemos conseguido experiencias lúdicas y docentes que ofrecen estrategias para generar sensaciones nuevas que provoquen el acercamiento social y educativo sobre los Objetivos de Desarrollo Sostenible 2030.

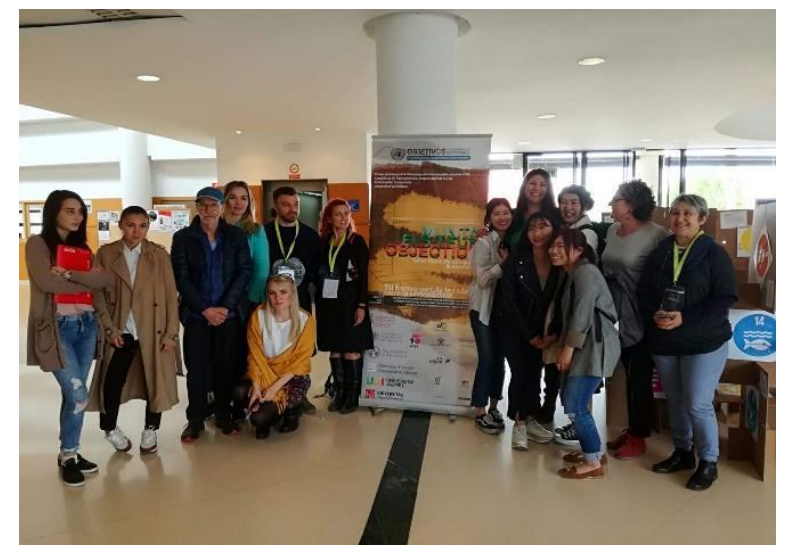

Fig. 10. 2019. Equipo de trabajo de Monta tus Objetivos 


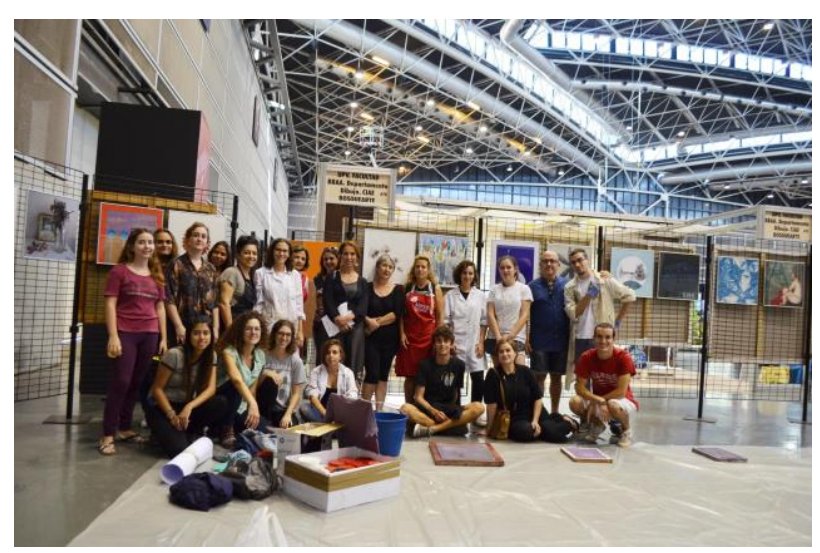

Fig. 11. 2018 noviembre. Equipo de trabajo y artistas

\section{Referentes}

United Nations Educational, Scientific and Cultural Organization. The Hangzhou Declaration

< http://unesdoc.unesco.org/images/0022/002212/221238m.pdf> [Consulta: 15 de marzo de 2018] http://www.upv.es/entidades/CIAE/, CIAE. Centro de Investigación en Arte y Entorno.

https://www.facebook.com/pg/Bosquearte/photos/?tab=album\&album_id=847034395358670, Bosquearte.

https://ecoeducacion.webs.upv.es/, DESEEEA

http://cropprotection.es/start/, Crop Protection

http://www.jardibotanic.org/, Jardibotanic

http://www.mapama.gob.es/es/ministerio/servicios/ayudas-subvenciones/programa-life/, Programa LIFE https://iberflora.feriavalencia.com/, IBERFLORA

https://www.horadelplaneta.es/, La hora del planeta WWF

http://reds-sdsn.es/, Red Española para el Desarrollo Sostenible

https://unsco.unmissions.org/, UNSCO

http://www.un.org/es/events/forestsday/

VÁZQUEZ GARCÍA, Lucía., (2018) “Emocionar, inspirar y educar a través del Arte”. Monográfico Arte y Sostenibilidad: impulsando una gestión cultural más sostenible, en Revista Conectando Audiencias. Ed. Asimétrica.

DE PISÓN, Eduardo., (2017) "Paisaje, cultura y sostenibilidad" (Ed. Acciona), en SMART: caminos hacia la sostenibilidad. Ed. Acciona.

NOVO, María., (2017) "El papel del arte y de la educación. Cambiar en tiempos de incertidumbre”, en SMART: caminos hacia la sostenibilidad. Ed. Acciona. 
SLAUGHTER, Sheila y Larry L. Leslie., (1997) Academic Capitalism: Politics, Policies, \& the Entrepreneurial University, Baltimore, The Johns Hopkins University Press, 276 pp. Citado en Ibarra Collado

The Hangzhou Declaration. Placing Culture at the Heart of Sustainable Development Policies. Adopted in Hangzhou, People's Republic of China, 17 Mayo de 2013

How can artists support the global transformation to sustainability?. Ed. IIASA, Vienna.

Long Horizons guide (reflections about art, artists and climate change). Ed. British Council \& Julie's Bicycle

La sostenibilidad en la Feria del Libro de Madrid, Antinomias, blog del sector del libro

MOOC | The Age of Sustainable Development - by Jeffrey Sachs | subt. en español

MOOC | Transforming our World - by Jeffrey Sachs | en inglés

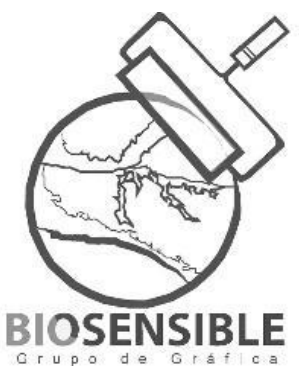

Este documento ha sido desarrollado por el Grupo de Gráfica Biosensible, Conformado por Ana Tomás Miralles, Tania Ansio Martínez y Rosángela Aguilar Briceño. 